Article

\title{
High-Sensitivity Determination of Nutrient Elements in Panax notoginseng by Laser-induced Breakdown Spectroscopy and Chemometric Methods
}

\author{
Tingting Shen ${ }^{1,+}{ }^{\dagger}$ Weijiao $\mathrm{Li}^{2,+}{ }^{+}$, Xi Zhang ${ }^{2}$, Wenwen Kong ${ }^{3}$, Fei Liu ${ }^{1,4, * \mathbb{D}}$, Wei Wang ${ }^{1}$ and \\ Jiyu Peng ${ }^{1}$ (1) \\ 1 College of Biosystems Engineering and Food Science, Zhejiang University, 866 Yuhangtang Road, \\ Hangzhou 310058, China; shentingtingstt@163.com (T.S.); 15236193955@163.com (W.W.); \\ jypeng@zju.edu.cn (J.P.) \\ 2 Chinese Materia Medica, Yunnan University of Chinese Medicine, Kunming 650500, China; \\ liweijiao163@163.com (W.L.); zhangxi1030@hotmail.com (X.Z.) \\ 3 School of Information Engineering, Zhejiang A \& F University, Hangzhou 311300, China; \\ wwkong16@zafu.edu.cn \\ 4 Key Laboratory of Spectroscopy Sensing, Ministry of Agriculture and Rural Affairs, Hangzhou 310058, China \\ * Correspondence: fliu@zju.edu.cn; Tel.: +86-571-88982825 \\ + The first two authors contribute equally to this paper.
}

Received: 20 March 2019; Accepted: 16 April 2019; Published: 18 April 2019

check for updates

\begin{abstract}
High-accuracy and fast detection of nutritive elements in traditional Chinese medicine Panax notoginseng (PN) is beneficial for providing useful assessment of the healthy alimentation and pharmaceutical value of PN herbs. Laser-induced breakdown spectroscopy (LIBS) was applied for high-accuracy and fast quantitative detection of six nutritive elements in PN samples from eight producing areas. More than 20,000 LIBS spectral variables were obtained to show elemental differences in PN samples. Univariate and multivariate calibrations were used to analyze the quantitative relationship between spectral variables and elements. Multivariate calibration based on full spectra and selected variables by the least absolute shrinkage and selection operator (Lasso) weights was used to compare the prediction ability of the partial least-squares regression (PLS), least-squares support vector machines (LS-SVM), and Lasso models. More than 90 emission lines for elements in PN were found and located. Univariate analysis was negatively interfered by matrix effects. For potassium, calcium, magnesium, zinc, and boron, LS-SVM models based on the selected variables obtained the best prediction performance with $R_{p}$ values of $0.9546,0.9176,0.9412,0.9665$, and 0.9569 and root mean squared error of prediction (RMSEP) of $0.7704 \mathrm{mg} / \mathrm{g}, 0.0712 \mathrm{mg} / \mathrm{g}, 0.1000 \mathrm{mg} / \mathrm{g}$, $0.0012 \mathrm{mg} / \mathrm{g}$, and $0.0008 \mathrm{mg} / \mathrm{g}$, respectively. For iron, the Lasso model based on full spectra obtained the best result with an $R_{p}$ value of 0.9348 and RMSEP of $0.0726 \mathrm{mg} / \mathrm{g}$. The results indicated that the LIBS technique coupled with proper multivariate chemometrics could be an accurate and fast method in the determination of PN nutritive elements for traditional Chinese medicine management and pharmaceutical analysis.
\end{abstract}

Keywords: Panax notoginseng; nutrient elements; laser-induced breakdown spectroscopy; least absolute shrinkage and selection operator; matrix effect; traditional Chinese medicine

\section{Introduction}

Panax notoginseng (commonly known as Sanqi or Tianqi) is a valuable Chinese herbal medicine that is in clinical trials or in clinical practice [1,2]. The U.S. Dietary Supplement Health and Education Act advocated Panax notoginseng (PN) as a dietary supplement in 1994 [3]. Modern research has shown 
that PN has biological activities in many aspects such as the blood system, cardiovascular system, nervous system, immune system, etc. [4]. Especially for heart and cerebrovascular diseases, PN can reduce myocardial oxygen consumption, improve myocardial ischemia, protect experimental cerebral ischemia, and is anti-heart rhythm [5,6]. PN also has good health functions such as lowering blood fat, lowering blood pressure, anti-shock, etc. [6].

Good health functions and medicinal value have increased the demand for PN in many countries. China is a major exporter of PN, and most of the PN comes from Yunnan Province [7]. The complexity of Yunnan's terrain makes PN's quality different in different producing areas [8]. The most obvious difference is nutrient elements in Panax notoginseng, because elemental content is directly related to the growth environment of the herbal medicine, as roots assimilate elements readily from the soil [9]. Nutritive elements in herbal medicine not only provide necessary nutrient elements for the human body [10], but also play significant roles in the formation of functional constituents such as Fe and $\mathrm{Zn}$, which are the active centers of some metalloenzymes [11-13]. Therefore, it is important to analyze the nutritive elements to control the quality of traditional Chinese medicine and that serve as the basis for pharmacological research.

Due to the importance of nutritive elements present in traditional Chinese medicine, common detection technologies including inductively-coupled plasma optical emission spectrometry (ICP-OES) [14], inductively-coupled plasma-mass spectrometry (ICP-MS) [15], and atomic absorption spectrometry (AAS) [16] were used to determine nutritive elements to analyze the value of herbs. Although the techniques above could obtain accurate results for elements, the samples must undergo complex and time-consuming pretreatments such as high temperature and high acid digestion [17]. These defects limit on-line and massive detection of nutritive elements in PN from different areas.

Laser-induced breakdown spectroscopy (LIBS) is a novel atomic technique that exhibits the advantages of fast analytical speed, multi-element analysis, minimal sample preparation, and high efficiency $[18,19]$. These attractive features have attracted researchers to provide laboratory analysis to explore the potential for LIBS to be a polyvalent monitoring and analysis tool for elements' detection [20], and especially nutritive elements in plant materials [21]. Braga et al. applied LIBS to analyze micronutrients in pellets of plant materials, and the results proved that LIBS technique combined with partial least-squares (PLS) is robust for elements determination [22]. Carvalho et al. compared femtosecond LIBS and nanosecond LIBS to profile nutrients in plant materials, and the result showed that the content values predicted by nanosecond LIBS multivariate modeling exhibit better agreement with reference mass fractions [23]. Those studies indicated that the LIBS technique has the potential to detect nutritive elements, and all mention that the "matrix effect" could affect the performance of the detection model. Matrix effects are related to the entire ablation/detection process and the ablation differences rooted in the chemical composition and physical properties of samples [24]. The composition of PN is complex, and the matrix effect is inevitable. He et al. detected soil nutrient elements potassium (K), calcium (Ca), magnesium $(\mathrm{Mg})$, iron (Fe), manganese (Mn), and sodium (Na) based on LIBS and found that the PLS model was the most suitable for quantitative analysis of elements [25]. The PLS method proved to be a robust model, mainly because PLS could reduce multicollinearity among variables and mitigate interference from the matrix effect. Other studies have also confirmed that SVM and Lasso are also good methods to build excellent regression models such as SVM models for quantitative analysis of $\mathrm{Cr}$ and Ni in iron alloys [26] and Lasso models for major element analysis of rocks [27].

Panax notoginseng in different producing areas has complex elemental compositions and structural differences. To the best of our knowledge, the quantitative detection of PN nutrient elements based on LIBS combined with chemometrics has not been investigated. The objective of this work is to explore fast and accurate quantitative detection of nutritive elements $\mathrm{K}, \mathrm{Ca}, \mathrm{Mg}$, Fe, zinc, and boron in PN samples from eight producing areas by laser-induced breakdown spectroscopy. LIBS spectra were used to analyze element differences and elemental fingerprinting in PN samples from different regions. Univariate calibration and multivariate calibration were employed to establish the calibration models for quantitative analysis of elements. Especially, PLS, SVM, and Lasso were compared to eliminate matrix effect interference and improve the sensitivity and stability of LIBS detection. 


\section{Materials and Methods}

\subsection{Panax notoginseng Samples}

All PN samples were obtained from 8 areas of Yunnan province in China, namely Xichou (1), Yongde (2), Malipo (3), Mile (4), Gejiu (5), Gengma (6), Shizong (7), and Qiubei (8). All samples were purchased in 2017 harvest seasons. Thirteen pieces of PN were sampled from the PN set of each origin randomly, and a total of $104 \mathrm{PN}$ samples was collected for further analysis. Each piece of PN was dried in an oven at $40{ }^{\circ} \mathrm{C}$ for approximately $3 \mathrm{~h}$ and was ground into powder by a tissue milling machine for consistent measurement. Two hundred milligrams of each PN powder were placed into a squared die set and pressed into a pellet with $700 \mathrm{MPa}$ of pressure for $30 \mathrm{~s}$. A total of 104 pellets was obtained, each of which was square with a 10-mm length for each side.

\subsection{Spectral Acquisition}

A self-assembled LIBS setup was used to realize LIBS spectral acquisition [28]. Laser pulses at $532 \mathrm{~nm}$ with maximum energy of $200 \mathrm{~mJ}$ and an 8-ns pulse width were generated by a Q-switched Nd:YAG pulse laser (Vlite 200, Beamtech, Beijing, China). The green laser passed through our self-made optical system and focused on $2 \mathrm{~mm}$ below one PN pellet's surface through a plano-convex lens ( $\mathrm{f}=100 \mathrm{~mm}$ ). The laser ablated $\mathrm{PN}$ and generated a high temperature plasma, which contained atoms, ions, electrons, and molecules. During out diffusion of plasma, each element in the plasma ionized to form continuous spectral lines. The information of elements was collected by a light collector and received by the spectrometer (ME5000, Andor Technology, Belfast, U.K.) combined with an ICCD camera (DH334T-18F-03, Andor Technology, Belfast, U.K.). The spectra between 230.77 and $883.24 \mathrm{~nm}$ with high resolution $(\lambda / \Delta \lambda=5000)$ were collected. A delay generator (DG645, Stanford Research Systems, Sunnyvale, CA, USA) was used to regulate the delay time between the ICCD camera and laser Q-switch that controlled the laser generation. The optimal experimental parameters were optimized with a laser energy of $60 \mathrm{~mJ}$, a delay time of $2.827 \mu \mathrm{s}$, and a gate width of $20 \mu \mathrm{s}$. Each PN pellet was placed on a sample stage of an $x-y-z$ motorized positioning system, which controlled the laser ablation path with $4 \times 4$ array. Therefore, $4 \times 4$ craters appeared on the pellet surface, and each crater had 5-times accumulation of laser pulses. The spectrum for each sample was recorded by the average of the 80 spectra $(4 \times 4 \times 5)$ to reduce fluctuation between the laser point-to-point. The interval distance of each hit was $2 \mathrm{~mm}$.

\subsection{Reference Method for Nutrient Elements' Content Determination}

The reference method for detecting nutrient elements $\mathrm{K}, \mathrm{Ca}, \mathrm{Mg}$, Fe, zinc ( $\mathrm{Zn}$ ), and boron (B) content in PN samples mainly relied on an inductively-coupled plasma optical emission spectrometer (ICP-OES) [29]. Before the determination by ICP-OES, the PN samples needed to experience microwave digestion and acid discharge pretreatment. Each pellet after LIBS acquisition was weighed and placed into modified polytetrafluoroethylene vessels with $5 \mathrm{~mL}$ of $65 \% \mathrm{HNO}_{3}$ and $1 \mathrm{~mL}$ of $30 \% \mathrm{H}_{2} \mathrm{O}_{2}$ for microwave digestion at $185^{\circ} \mathrm{C}$. Then, modified polytetrafluoroethylene vessels with digested liquid were placed in a $165^{\circ} \mathrm{C}$ furnace to discharge the acid till a drop of fuming digested liquid remained. The acid elimination pretreatment happened in the fuming cupboard. The least digested liquid was diluted to a volume of $30 \mathrm{~mL}$ with high-purity water by the weighing method. The final dilution was applied to detect elemental content by ICP-OES.

\subsection{Data Analysis}

\subsubsection{Data Preprocessing}

For remitting systematic and random errors during the experiment, wavelet transform (WT) was used to preprocess the raw spectral. As an efficient denoising method, WT uses a set of wavelet basis functions to remove invalid information (noise) and reserve valid sharp peaks or spikes [30]. Daubechies 8 with the decomposition scale of 3 was selected in our paper. After WT preprocessing, the 
spectral of $104 \mathrm{PN}$ samples were sorted from the lowest to highest according to $\mathrm{K}, \mathrm{Ca}, \mathrm{Mg}, \mathrm{Fe}, \mathrm{Zn}$, and B content values (by ICO-OES), respectively. The Kennard-Stone (KS) algorithm [29], which could avoid bias in sample selection, was used to split 104 PN samples into a calibration set and a validation set. Seventy two PN samples were selected for the calibration set, while the remaining 32 samples formed the prediction set. Univariate analysis and multivariate analysis were initially done using PN samples in the calibration set.

\subsubsection{Multivariate Analysis Methods}

Multivariate analysis employed PLS, SVM, and Lasso to establish calibration models, and the parameter selection of models was done using cross-validation. Lasso was used to select effective variables to improve multivariate analysis performance.

Partial least-squares regression (PLS) is an established analytical tool for relating multivariate data analysis [31]. A PLS model expresses the relation between LIBS spectral information X and the content of element $\mathrm{Y}$ in PN samples. Spectral matrix $\mathrm{X}$ and content matrix $\mathrm{Y}$ were decomposed, and the invalid information in the two matrixes was handled simultaneously. Principal component information was calculated after matrix decomposition. When calculating the principal component, PLS considered larger variance of the principal component to extract more useful information and also made the principal component (latent variables (LVs)) and element concentration Y more relevant to maximize the linear relationship between spectral variables and concentrations [32,33]. The five-fold cross-validation procedure was used, and the number of LVs was selected when the first minimum or the knee point for the root mean squared error of cross-validation (RMSECV) in calibration set vs. LVs curve was obtained.

Least-squares support vector machine (LS-SVM) is an improvement of the standard SVM based on the structural risk minimization (SRM) approach proposed by Vapnik et al. [34]. LS-SVM used the least-squares linear system as the loss function and applied a set of linear equations to replace the complicated quadratic programming method, which was adopted by the standard SVM. LS-SVM reduces the computational complexity and solution speeds and improves generalization ability of model [35]. Compared with PLS, LS-SVM could not only solve linear relation, but also nonlinear regression problems. The radial basis function (RBF) kernel function and five-fold cross-validation were utilized to establish the LS-SVM calibration model. The penalty parameters (c) and kernel function parameters (g) of LS-SVM were optimized by a grid-search procedure in the range of 103-1010, and the best $c$ and $g$ were determined with the minimal value of RMSECV.

The least absolute shrinkage and selection operator (Lasso) is a penalized shrunken regression method [36]. Lasso is also a dimensionality reduction method for both linear and nonlinear cases. The penalty method L1 was introduced when spectral matrix X did not belong to column full rank. Lasso selected variables from spectral data according to the penalty method L1 [27]. The penalty method L1 compressed the original coefficients $\beta$, and some original small coefficients were directly compressed to 0 . The variables corresponding to these $\beta=0$ were regarded as non-significant variables to be discarded directly. The penalty method took the value when the penalty likelihood function was the smallest as the estimated value of the regression coefficient [27,37]. Five-fold cross-validation was also applied to establish the Lasso calibration model and confirm the best model parameter the boundary value $t$, which is analogous to LVs for PLS and the number of non-zero $\beta$, which is expressed as either $t$ in the optimization equation or the number of steps in the step-wise procedure.

\subsubsection{Performance Evaluation}

The correlation coefficient (R), RMSECV, and root mean squared error of the prediction set (RMSEP) were used to evaluate the performance of the quantitative models for nutritive elements' content detection [38]. Correlation coefficient (R) means correlation between target element content obtained by ICP-OES and target element content detected by LIBS. $R_{c}$ is correlation coefficient of the calibration set and $R_{p}$ is the correlation coefficient of the prediction set. The closer the $\mathrm{R}$ value is to 1 
with the smaller root mean squared error, the better the performance and detectability of LIBS variables and calibration models.

\subsubsection{Software Tools}

LIBS spectra acquisition was carried out by Andor SOLIS for Imaging (v4.26, Andor Technology, Belfast, U.K.). Data analysis was executed by MATLAB R2017a (The MathWorks, Inc., Natick, MA, USA). Origin Pro 2015 (Origin Lab Corporation, Northampton, MA, USA) was applied for graphs' design.

\section{Results}

\subsection{Nutritive Elements Content of Panax Notoginseng}

Nutritive elements $\mathrm{K}, \mathrm{Ca}, \mathrm{Mg}, \mathrm{Fe}, \mathrm{Zn}$, and B content of eight producing areas detected by ICP-OES are shown in Table 1. For PN samples from all regions, the content of B and Zn was much smaller than that of Fe, which also belonged to the microelement. Commonly belonging to nutritive elements, the content of $\mathrm{K}$ was nearly ten-times that of $\mathrm{Ca}$ and $\mathrm{Mg}$ in all PN samples. The $\mathrm{K}, \mathrm{Ca}, \mathrm{Mg}, \mathrm{Fe}$, and $\mathrm{Zn}$ content of PN in Malipo (Group 3) was higher than the other regions, and the B content was second only to the PN in Xichou (Group 1), which indicated that the quality of PN in Malipo is best. Compared with other regions, the content of those elements in PN samples from Gejiu (Group 5) and Gengma (Group 6) was relatively small. The content of these nutritive elements could reflect the quality of PN goods from different producing areas. Undoubtedly, the method of rapid and simultaneous detection of elements content is conducive to the regulation of the PN commodity market.

Table 1. Nutritive elements content (mg/g) of Panax notoginseng.

\begin{tabular}{|c|c|c|c|c|c|c|c|c|c|}
\hline \multirow{2}{*}{ Element } & Groups $^{a}$ & 1 & 2 & 3 & 4 & 5 & 6 & 7 & 8 \\
\hline & Number & 13 & 13 & 13 & 13 & 13 & 13 & 13 & 13 \\
\hline \multirow{4}{*}{ K } & Min & 11.5682 & 8.2990 & 12.1523 & 11.0964 & 7.8709 & 6.4402 & 10.2981 & 8.7456 \\
\hline & Max & 17.4899 & 15.4417 & 19.7162 & 18.3589 & 10.8974 & 13.8608 & 15.8312 & 18.0112 \\
\hline & Mean & 14.0558 & 11.7255 & 16.0186 & 14.7329 & 9.3546 & 10.0683 & 12.8228 & 13.9661 \\
\hline & S.D. & 1.6676 & 1.9894 & 2.5840 & 2.0317 & 0.9795 & 1.8793 & 1.4315 & 2.71071 \\
\hline \multirow{4}{*}{$\mathrm{Ca}$} & Min & 1.4194 & 1.1814 & 1.5225 & 1.2717 & 1.2216 & 1.0807 & 1.0997 & 1.4007 \\
\hline & Max & 2.1659 & 1.8081 & 2.4316 & 2.3112 & 1.8509 & 2.3226 & 1.9342 & 2.3260 \\
\hline & Mean & 1.7756 & 1.4200 & 1.9667 & 1.6736 & 1.4758 & 1.3450 & 1.4921 & 1.8509 \\
\hline & S.D. & 0.2292 & 0.2062 & 0.4276 & 0.3137 & 0.1993 & 0.3683 & 0.2203 & 0.4987 \\
\hline \multirow{4}{*}{$\mathrm{Mg}$} & Min & 0.8821 & 0.8153 & 1.0908 & 1.1779 & 1.0813 & 0.5774 & 0.9143 & 1.1373 \\
\hline & Max & 1.2918 & 1.4632 & 1.9072 & 1.6739 & 1.7435 & 1.1640 & 1.8240 & 1.8797 \\
\hline & Mean & 1.1343 & 1.0583 & 1.5977 & 1.4081 & 1.3567 & 0.7984 & 1.2961 & 1.3825 \\
\hline & S.D. & 0.1194 & 0.1824 & 0.2170 & 0.1559 & 0.2113 & 0.1802 & 0.2730 & 0.2240 \\
\hline \multirow{4}{*}{$\mathrm{Fe}$} & Min & 0.0288 & 0.0711 & 0.0837 & 0.1003 & 0.0661 & 0.0781 & 0.1154 & 0.0783 \\
\hline & Max & 0.8145 & 0.3023 & 1.0317 & 0.9004 & 0.3862 & 0.5021 & 0.7329 & 0.7258 \\
\hline & Mean & 0.2401 & 0.1598 & 0.5550 & 0.4918 & 0.1885 & 0.1903 & 0.3003 & 0.3785 \\
\hline & S.D. & 0.0938 & 0.0668 & 0.1039 & 0.0546 & 0.0869 & 0.0779 & 0.0803 & 0.0840 \\
\hline \multirow{4}{*}{$\mathrm{Zn}$} & Min & 0.0147 & 0.0075 & 0.0122 & 0.0116 & 0.0103 & 0.0085 & 0.0069 & 0.0121 \\
\hline & Max & 0.0351 & 0.0225 & 0.0303 & 0.0250 & 0.0159 & 0.0217 & 0.0159 & 0.0242 \\
\hline & Mean & 0.0203 & 0.0130 & 0.0213 & 0.0192 & 0.0129 & 0.0131 & 0.0113 & 0.0183 \\
\hline & S.D. & 0.0073 & 0.0037 & 0.0089 & 0.0044 & 0.0016 & 0.0041 & 0.0022 & 0.0041 \\
\hline \multirow{4}{*}{ B } & Min & 0.0091 & 0.0061 & 0.0057 & 0.0035 & 0.0038 & 0.0027 & 0.0074 & 0.0047 \\
\hline & Max & 0.0154 & 0.0148 & 0.0165 & 0.0159 & 0.0134 & 0.0167 & 0.0147 & 0.0156 \\
\hline & Mean & 0.0138 & 0.0105 & 0.0131 & 0.0130 & 0.0074 & 0.0079 & 0.0105 & 0.0132 \\
\hline & S.D. & 0.0032 & 0.0024 & 0.0066 & 0.0057 & 0.0026 & 0.0036 & 0.0023 & 0.0038 \\
\hline
\end{tabular}

a 1: Xichou, 2: Yongde, 3: Malipo, 4: Mile, 5: Gejiu, 6: Gengma, 7: Shizong, 8: Qiubei. 


\subsection{LIBS Spectra Analysis}

The average spectrum of each area in the range of $230.77-883.24 \mathrm{~nm}$ is shown in Figure 1. The LIBS spectra of PN samples from the eight areas were observed to have a similar tendency, which signified that PN in different producing areas had the same element species and similar matrix compositions. Molecule bands $\mathrm{CN}$ and $\mathrm{H}$ and atomic line O I, which commonly appeared in the LIBS spectra of organic samples, can be observed in Figure 1. The emission lines of $\mathrm{K}, \mathrm{Ca}, \mathrm{Mg}$, and $\mathrm{Fe}$ in eight areas of PN samples are also obvious in Figure 2 with different intensity values such as PN in Gengma (Group 6) having the lowest Mg emission line and PN in Malipo (Group 3) having the highest Mg emission line, which is consistent with the results in Table 1 . The intensity difference of element emission lines indicated that different habitats significantly changed the content of PN and formed a specific proportion of elements.

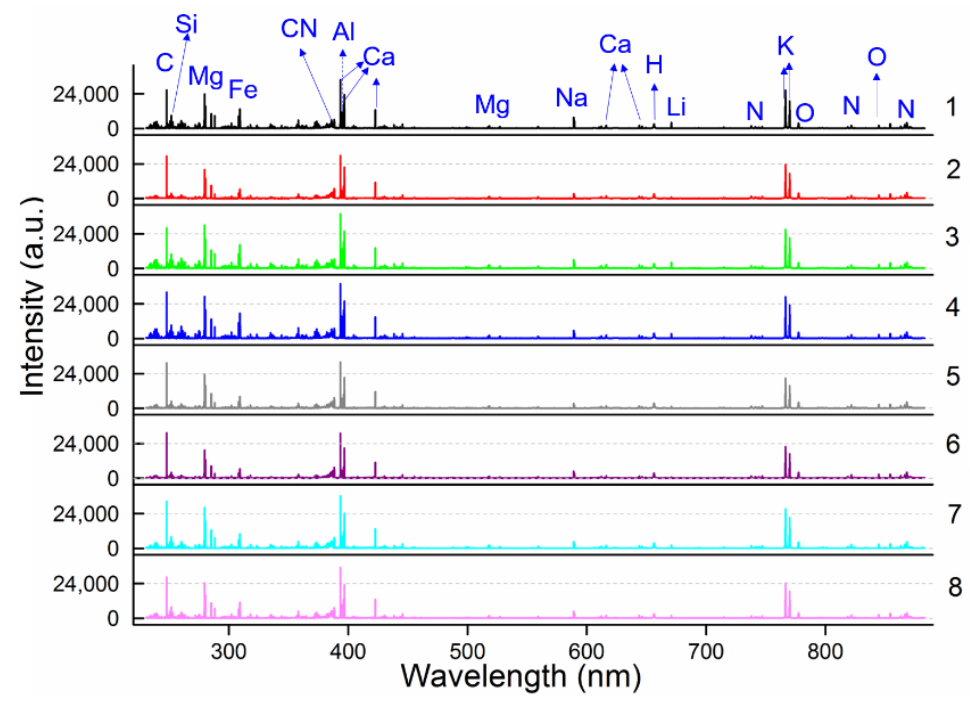

Figure 1. The average spectrum of each area in the range of $230.77-883.24 \mathrm{~nm}, 1:$ Xichou, 2: Yongde, 3 : Malipo, 4: Mile, 5: Gejiu, 6: Gengma, 7: Shizong, 8: Qiubei.
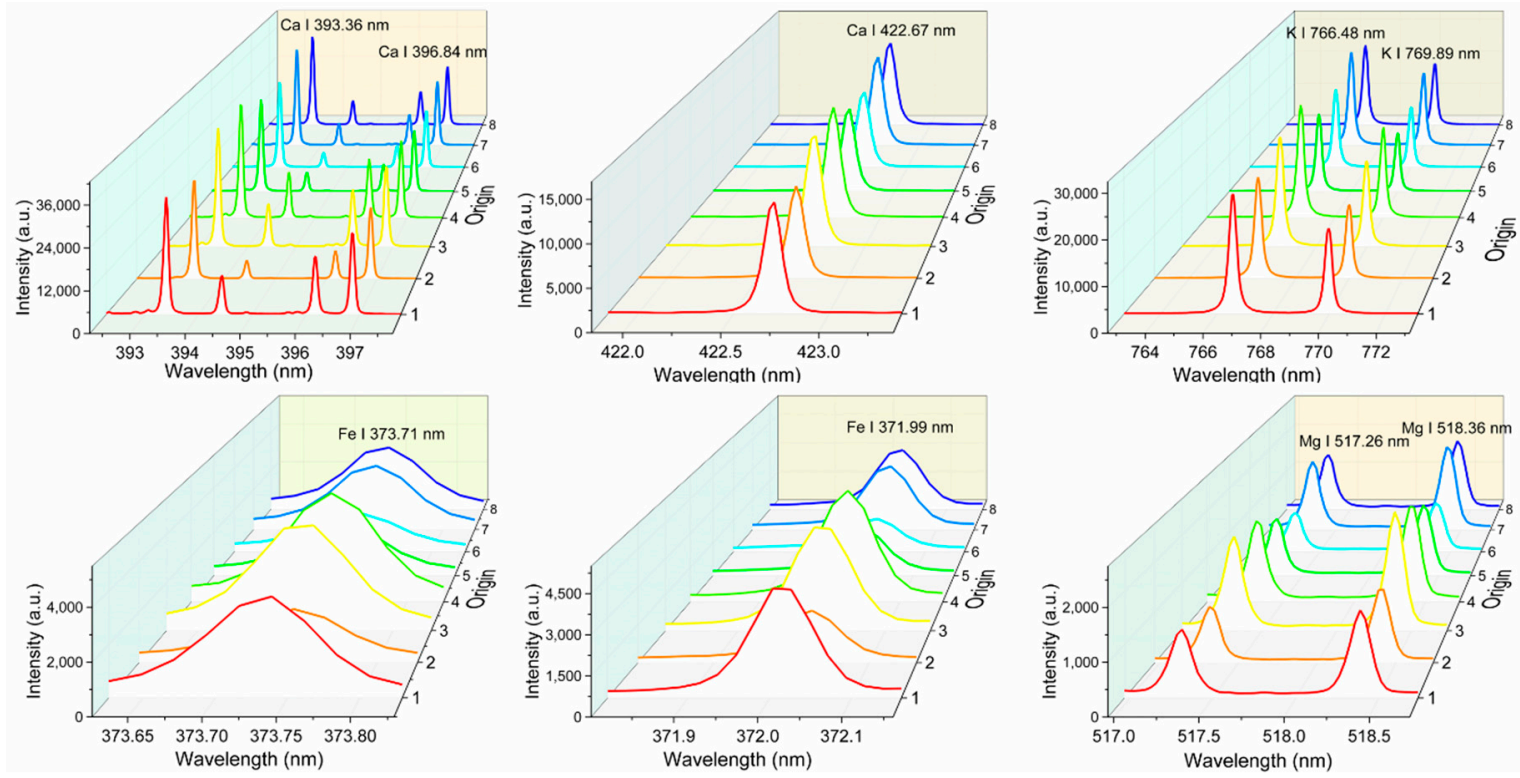

Figure 2. The emission lines of $\mathrm{K}, \mathrm{Ca}, \mathrm{Fe}$ and $\mathrm{Mg}$ for univariate analysis.

Based on the Kurucz database and the National Institute of Standards and Technology (NIST) Atomic Spectra Database (ASD), more than 90 emission lines whose intensity values were above 1000 
a.u. were verified identities, and the elements are shown in Table 2. Nutritive element Ca had 35 emission lines, and Ca II $393.37 \mathrm{~nm}$ had the maximum intensity value of all element emission lines in the PN samples. The emission line for carbon $(C)$ without the highest intensity value may be due to the relatively long delay time, since the intensity of emission lines changed quickly within the time [18]. The observed emission lines for oxygen and nitrogen may come from the common contribution of the PN sample and air. Table 2 presents the capability of LIBS technique to detect different elements from PN samples.

Table 2. The obvious spectral emission lines of Panax notoginseng (PN) based on the NIST database.

\begin{tabular}{cc}
\hline Elements & Wavelength (nm) \\
\hline C I & $247.86,296.72$ \\
Si I & $250.68,251.43,251.61,251.92,252.41,288.15$ \\
Fe I & $302.06,371.99,385.99,293.69,498.24,499.41$ \\
Fe II & $253.54,257.60,259.37,260.54,263.08$, \\
Mg I & $279.55,279.80,280.27$ \\
Mg II & $277.98,382.94,383.23,383.83,389.19,516.73,517.27,518.36$ \\
Ca I & $299.50,300.09,300.69,422.67,428.30,428.94,429.90,430.25,430.77,431.87,442.54,443.50,458.15,458.60,527.03$, \\
Ca II & $558.87,559.45,559.85,585.75,610.27,612.22,616.22,643.91,644.98,646.26,649.38,671.77,714.82,854.21$ \\
Sc II & $315.89,317.93,373.69,393.37,396.85,866.21$ \\
CN & 364.37 \\
Al I & $394.40,396.15$ \\
K I & $693.87,766.49,769.90$ \\
Sr I & 460.73 \\
Sr II & $407.77,421.55$ \\
Na I & $589.00,589.59$ \\
H & 656.28 \\
O I & $777.42,844.67$ \\
Li I & 670.79 \\
N I & $385.01(\mathrm{CN} 4-4), 385.44(\mathrm{CN} 3-3), 386.15(\mathrm{CN} 2-2), 387.12(\mathrm{CN} 1-1), 388.32(\mathrm{CN} 0-0)$ \\
\hline
\end{tabular}

\subsection{Univariate Analysis}

Univariate analysis is a calibration curve method that reflects that the intensity of emission line is proportional to the content of the target element in samples [39]. In common LIBS quantitative analysis, sensitive emission lines of the target element without self-absorption and overlapping peaks are the preferred choice for univariate analysis [18]. According to the NIST database, the sensitive emission lines K I 766.49 nm, K I 769.90 nm, Ca II 393.37 nm, Ca II 396.85 nm, Ca I 422.67 nm, Mg I $517.27 \mathrm{~nm}, \mathrm{Mg}$ I $518.36 \mathrm{~nm}$, Fe I $373.71 \mathrm{~nm}$, and Fe I $371.99 \mathrm{~nm}$ were selected to build univariate analysis models for Panax notoginseng. As shown in Figure 2, the emission lines of these nutritive elements are smooth without interference peaks. The peak intensity of $\mathrm{K}, \mathrm{Ca}, \mathrm{Mg}$, and Fe in $\mathrm{Groups} 2$, 5 , and 6 was significantly lower than other groups, which is consistent with the difference in element content in Table 1. Because of the low content, the sensitive emission lines of B and Zn were difficult to discriminate for univariate analysis.

The univariable calibration and prediction results of the above selected emission lines of nutritive elements are shown in Table 3. For univariate analysis of K content, K I $769.90 \mathrm{~nm}$ performed better than K I $766.49 \mathrm{~nm}$, with $R_{c}$ of 0.8413 , RMSECV of $1.370 \mathrm{mg} / \mathrm{g}$ in calibration, and $R_{p}$ of $0.7836, \mathrm{RMSCP}$ of $1.610 \mathrm{mg} / \mathrm{g}$ in prediction. The results of Ca I $422.67 \mathrm{~nm}, \mathrm{Mg}$ I $517.27 \mathrm{~nm}$, and Fe I $371.99 \mathrm{~nm}$ were all better than the other same element emission lines. Overall, the univariate analysis model for Fe I $371.99 \mathrm{~nm}$ performed best with $R_{c}$ of 0.8944 , RMSECV of $0.082 \mathrm{mg} / \mathrm{g}$ in calibration and $R_{p}$ of 0.8577 , RMSCP of $0.097 \mathrm{mg} / \mathrm{g}$ in prediction. However, the performance of univariate analysis in Table 2 is not sufficient to generate a robust and accurate predictive model for the content of $\mathrm{K}, \mathrm{Ca}, \mathrm{Mg}$, and Fe in PN samples. 
Table 3. The obvious spectral emission lines of PN based on the NIST database.

\begin{tabular}{ccccc}
\hline \multirow{2}{*}{$\begin{array}{c}\text { Emission Lines } \\
\text { nm }\end{array}$} & $\boldsymbol{R}_{\boldsymbol{c}}$ & RMSECV $\mathbf{~ m g / g}$ & $\boldsymbol{R}_{\boldsymbol{p}}$ & RMSEP $\mathbf{~ m g / g}$ \\
\cline { 2 - 5 } & 0.8324 & 1.4002 & 0.7476 & 1.7601 \\
K I 766.49 & 0.8413 & 1.3707 & 0.7836 & 1.6103 \\
K I 769.90 & 0.6872 & 0.1284 & 0.6327 & 0.1282 \\
Ca II 393.37 & 0.7764 & 0.1104 & 0.7118 & 0.1160 \\
Ca II 396.85 & 0.7941 & 0.1062 & 0.7779 & 0.1074 \\
Ca I 422.67 & 0.8403 & 0.1519 & 0.7564 & 0.1927 \\
Mg I 517.27 & 0.7520 & 0.1840 & 0.7168 & 0.2025 \\
Mg I 518.36 & 0.7509 & 0.1217 & 0.8378 & 0.1027 \\
Fe I 373.71 & 0.8944 & 0.0820 & 0.8577 & 0.0973 \\
Fe I 371.99 & & & &
\end{tabular}

\subsection{Multivariate Analysis}

Multivariate analysis attempted to analyze the relationship between more variables of LIBS spectra and nutrient elements $\mathrm{K}, \mathrm{Ca}, \mathrm{Mg}, \mathrm{Fe}, \mathrm{Zn}$, and B content obtained by ICP-OES. Full spectra or several selected variables combined with chemometric methods PLS, LS-SVM and Lasso were used to establish calibration models for the objective nutritive elements content.

\subsubsection{Modeling Using Full Spectra}

The results for multivariate analysis based on full spectra from $230.77 \mathrm{~nm}-883.24 \mathrm{~nm}$ are shown in Table 4. For K and $\mathrm{Ca}$, the multivariate analysis by Lasso and PLS achieved good performance with $R_{p}$ higher than 0.9500 . For $\mathrm{Mg}$ and $\mathrm{Fe}$, the multivariate analysis based on full spectra by Lasso achieved the best performance with $R_{p}$ values of 0.9207 and 0.9348 and RMSEP of $0.7740 \mathrm{mg} / \mathrm{g}$ and $0.0722 \mathrm{mg} / \mathrm{g}$, respectively. For Zn and B, PLS models obtained the best performance with $R_{p}$ values of 0.9460 and 0.9475 and RMSEP of $0.0016 \mathrm{mg} / \mathrm{g}$ and $0.0010 \mathrm{mg} / \mathrm{g}$, respectively.

Table 4. The results for multivariate analysis based on full spectra (22,036 variables) by PLS, LS-SVM, and least absolute shrinkage and selection operator (Lasso). RMSEP, root mean squared error of prediction.

\begin{tabular}{|c|c|c|c|c|c|c|}
\hline \multirow{2}{*}{ Element } & \multirow{2}{*}{ Model } & \multirow{2}{*}{ Parameter } & \multicolumn{2}{|c|}{ Calibration Set } & \multicolumn{2}{|c|}{ Prediction Set } \\
\hline & & & $R_{c}$ & RMSECV mg/g & $R_{p}$ & RMSEP mg/g \\
\hline \multirow{3}{*}{ K } & PLS $^{d}$ & $10^{\mathrm{a}}$ & 0.9558 & 0.8120 & 0.9505 & 0.7152 \\
\hline & LS-SVM & $(992.5,799,024.9)^{b}$ & 0.9800 & 0.3120 & 0.9391 & 0.8721 \\
\hline & Lasso & $53^{c}$ & 0.9547 & 0.7740 & 0.9496 & 0.7956 \\
\hline \multirow{3}{*}{$\mathrm{Ca}$} & PLS $^{d}$ & $13^{a}$ & 0.9563 & 0.0868 & 0.9513 & 0.0722 \\
\hline & LS-SVM & $(111.5,16,929,970)^{b}$ & 0.9799 & 0.0357 & 0.9135 & 0.1101 \\
\hline & Lasso & $54^{c}$ & 0.9533 & 0.0872 & 0.9508 & 0.0798 \\
\hline \multirow{3}{*}{$\mathrm{Mg}$} & PLS & $11^{\mathrm{a}}$ & 0.9270 & 0.1066 & 0.9171 & 0.1182 \\
\hline & LS-SVM & $(236.1,344,300.9)^{b}$ & 0.9601 & 0.0986 & 0.9011 & 0.1246 \\
\hline & Lasso $^{\mathrm{d}}$ & $51^{c}$ & 0.9294 & 0.1022 & 0.9207 & 0.1110 \\
\hline \multirow{3}{*}{$\mathrm{Fe}$} & PLS & $4^{a}$ & 0.9234 & 0.0791 & 0.9334 & 0.0906 \\
\hline & LS-SVM & $(311.9,4,680,480)^{b}$ & 0.9799 & 0.0451 & 0.9284 & 0.0854 \\
\hline & Lasso $^{d}$ & $51^{\mathrm{c}}$ & 0.9506 & 0.0549 & 0.9348 & 0.0762 \\
\hline \multirow{3}{*}{$\mathrm{Zn}$} & PLS $^{d}$ & $4^{\mathrm{a}}$ & 0.9503 & 0.0017 & 0.9460 & 0.0016 \\
\hline & LS-SVM & $(289.3,1,593,133.6)^{b}$ & 0.9886 & 0.0009 & 0.9060 & 0.0021 \\
\hline & Lasso & $54^{c}$ & 0.9406 & 0.0015 & 0.9228 & 0.0019 \\
\hline \multirow{3}{*}{ B } & $\mathrm{PLS}^{\mathrm{d}}$ & $4^{a}$ & 0.9566 & 0.0008 & 0.9475 & 0.0010 \\
\hline & LS-SVM & $(244.1,48,672.5)^{b}$ & 0.9866 & 0.0007 & 0.9036 & 0.0014 \\
\hline & Lasso & $52^{c}$ & 0.9502 & 0.0009 & 0.9348 & 0.0009 \\
\hline
\end{tabular}

${ }^{a}$ is the parameter for PLS for the number of latent variables (LVs), ${ }^{b}$ is the parameter for LS-SVM for penalty parameters (c) and kernel function parameters $(\mathrm{g}) ;{ }^{\mathrm{c}}$ is the parameter for parameter for Lasso for the boundary value $\mathrm{t}$; $\mathrm{d}$ means the best prediction model among three quantitative analysis methods for the specific element. 
For all the target elements in the PN samples, PLS and Lasso analysis performed well in multivariate analysis based on LIBS full spectra, which may be because PLS and Lasso were more suitable for solving multi-collinearity problems, making full use of the useful element information from the 22,036 variables for relationship fitting between LIBS and element content. LS-SVM performed well in calibration sets for all tested elements, but poor in prediction sets, which means over-fitting phenomenon occurred. A huge difference in the number of variables and the number of samples may cause this phenomenon.

\subsubsection{Modeling Using Selected Variables}

During the Lasso modeling process, irrelevant or insignificant coefficients of corresponding variables were penalized to zero and discarded directly [40]. Therefore, relevant or significant variables could be selected by comparing regression coefficients (weights) for each variable. The weights plot of the Lasso models for the nutrient elements are shown in Figure 3.
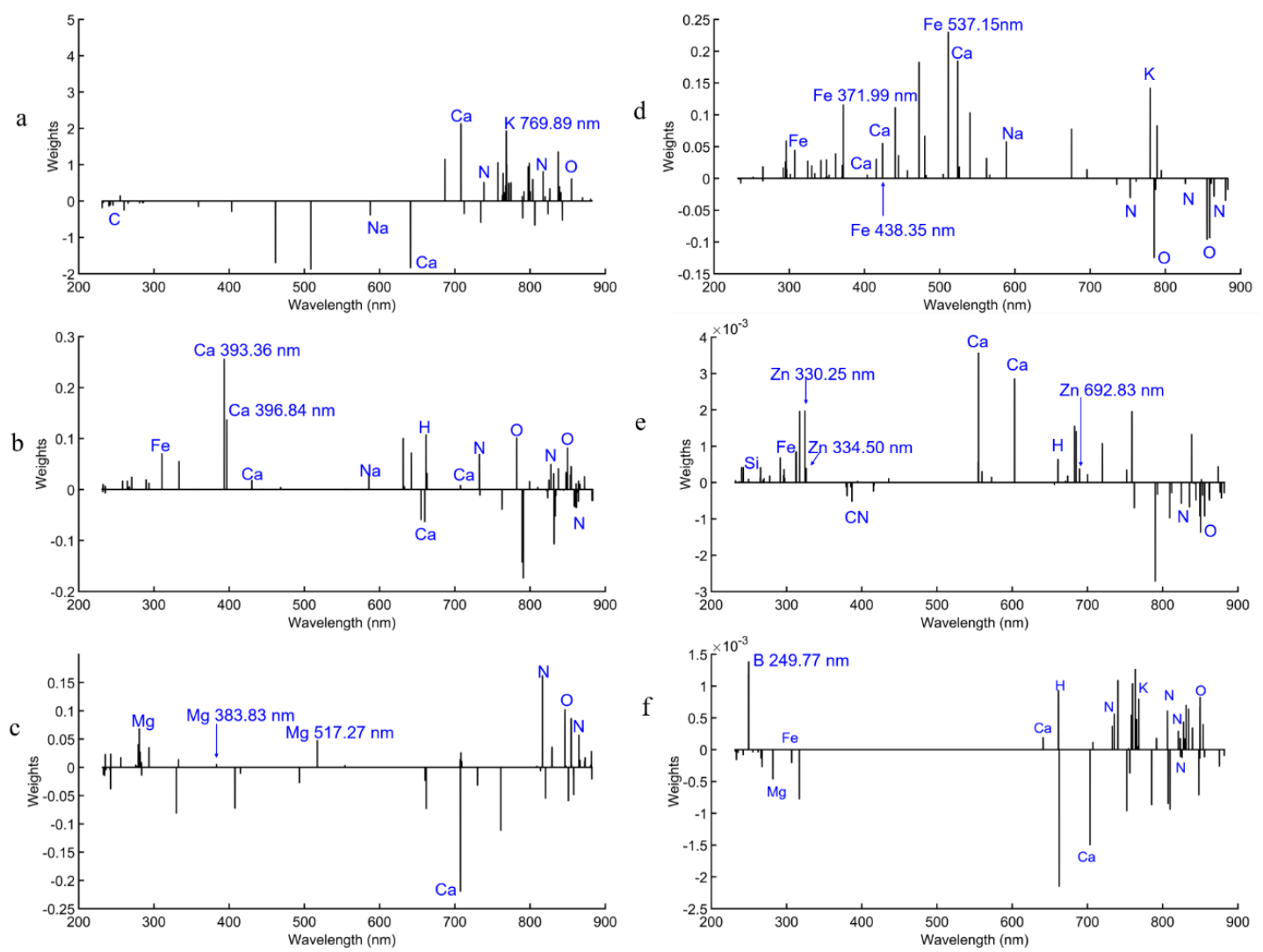

Figure 3. Weights plot of Lasso models for the nutrient elements $\mathrm{K}(\mathbf{a}), \mathrm{Ca}(\mathbf{b}), \mathrm{Mg}(\mathbf{c}), \mathrm{Fe}(\mathrm{d}), \mathrm{Zn}(\mathbf{e})$, and $B(\mathbf{f})$.

In the case of $\mathrm{K}$, the emission line at $769.89 \mathrm{~nm}$ showed strong correlation to the concentration. Ca II $393.36 \mathrm{~nm}$ and Ca II 396.84 showed strong correlation to the Ca concentration; Mg I $517.27 \mathrm{~nm}$ and Mg I $383.83 \mathrm{~nm}$ showed correlation to Mg concentration; Fe I $371.99 \mathrm{~nm}$, Fe I $438.35 \mathrm{~nm}$, and Fe I $537.15 \mathrm{~nm}$ showed strong correlation to Fe concentration; Zn I $330.25 \mathrm{~nm}$, Zn I $334.50 \mathrm{~nm}$, and Zn I $692.83 \mathrm{~nm}$ showed strong correlation to $\mathrm{Zn}$ concentration; B I $249.77 \mathrm{~nm}$ showed strong correlation to B concentration. It is clear that other multiple lines such as $\mathrm{N}, \mathrm{O}, \mathrm{H}$, and $\mathrm{Ca}$ exist in the weight plots of each element (Figure 3), which is due to the complex matrix components of $\mathrm{PN}$ and random noise. There were high weights of multiple lines such as $\mathrm{N}$ and $\mathrm{O}$ in $\mathrm{K}, \mathrm{Ca}, \mathrm{Mg}, \mathrm{Zn}$, and $\mathrm{B}$, which indicated that these elements contributed significantly to quantitative analysis of the target elements, and the interactions among all the elements in PN herbs could not be ignored. The target elements may combine with $\mathrm{N}$ and $\mathrm{O}$ to compose compounds such as aerobic compounds or amino acids. The multiple 
lines like $\mathrm{N}$ and $\mathrm{O}$ may also come from air ablation and reflect noise from environmental fluctuation. Compared with the spectrum of one PN sample (Figure 3d,h), we also found that background signals without emission lines were also contributing to the quantification study of nutrient elements such as the unmarked weight lines in the Lasso weights plot for $\mathrm{Mg}$ (Figure 3c). The background information with high weights may be related to the matrix effects of the PN samples.

The LIBS variables with non-zero weights values in Lasso models were selected for multivariate analysis by PLS, LS-SVM, and Lasso, and the results are shown in Table 5. The number of LIBS variables selected by Lasso for $\mathrm{K}, \mathrm{Ca}, \mathrm{Mg}$, Fe, $\mathrm{Zn}$, and B was 64, 73, 61, 66, 73, and 62, respectively. For $\mathrm{K}, \mathrm{Ca}, \mathrm{Mg}, \mathrm{Zn}$, and B content predictions, PL-SVM models based on the selected variables by Lasso achieved the best performance with $R_{p}$ values of $0.9546,0.9176,0.9412,0.9665$, and 0.9569 and RMSEP of $0.7704 \mathrm{mg} / \mathrm{g}, 0.0712 \mathrm{mg} / \mathrm{g}, 0.1000 \mathrm{mg} / \mathrm{g}, 0.0012 \mathrm{mg} / \mathrm{g}$, and $0.0008 \mathrm{mg} / \mathrm{g}$, respectively. For Fe content predictions, PLS models achieved the best performance with a $R_{p}$ value of 0.9169 and an RMSEP of $0.0724 \mathrm{mg} / \mathrm{g}$. On the whole, LS-SVM models could effectively predict the content of elements, followed by PLS models. Lasso quantitative analysis based on the selected variables preformed worse than the other analysis methods based on the same variables and Lasso quantitative analysis based on full spectra with 22,036 variables. This is because Lasso quantitative analysis based on the selected variables may lose some valid variables after over-screening these variables by the penalty method $L_{1}$.

Table 5. The results for multivariate analysis based on the selected variables by PLS, LS-SVM, and Lasso.

\begin{tabular}{|c|c|c|c|c|c|c|}
\hline \multirow{2}{*}{$\begin{array}{l}\text { Element } \\
\text { (number) }\end{array}$} & \multirow{2}{*}{ Model } & \multirow{2}{*}{ Parameter } & \multicolumn{2}{|c|}{ Calibration Set } & \multicolumn{2}{|c|}{ Prediction Set } \\
\hline & & & $R_{c}$ & RMSECV mg/g & $R_{p}$ & RMSEP mg/g \\
\hline \multirow{3}{*}{ K (64) } & PLS & $8^{a}$ & 0.9655 & 0.6852 & 0.9530 & 0.7853 \\
\hline & LS-SVM $^{\mathrm{d}}$ & $(192.1,6,274.1)^{b}$ & 0.9894 & 0.3864 & 0.9546 & 0.7704 \\
\hline & Lasso & $78^{\mathrm{c}}$ & 0.9689 & 0.6491 & 0.9482 & 0.8239 \\
\hline \multirow{3}{*}{$\mathrm{Ca}(73)$} & PLS & $13^{a}$ & 0.9420 & 0.0638 & 0.9047 & 0.0757 \\
\hline & LS-SVM $^{\mathrm{d}}$ & $(691.7,19,536.2)^{b}$ & 0.9890 & 0.0299 & 0.9176 & 0.0712 \\
\hline & Lasso & $66^{\mathrm{c}}$ & 0.9416 & 0.0639 & 0.9012 & 0.0776 \\
\hline \multirow{3}{*}{$\operatorname{Mg}(61)$} & PLS & $7^{a}$ & 0.9405 & 0.0957 & 0.9365 & 0.0979 \\
\hline & LS-SVM $^{\mathrm{d}}$ & $(146.2,3,195.7)^{b}$ & 0.9833 & 0.0053 & 0.9412 & 0.1000 \\
\hline & Lasso & $60^{c}$ & 0.9236 & 0.1080 & 0.9291 & 0.1034 \\
\hline \multirow{3}{*}{ Fe (66) } & PLS $^{d}$ & $6^{a}$ & 0.9299 & 0.0684 & 0.9169 & 0.0724 \\
\hline & LS-SVM & $(2585.9,20,694.3)^{b}$ & 0.9999 & 0.0002 & 0.9159 & 0.0891 \\
\hline & Lasso & $100^{c}$ & 0.9070 & 0.0784 & 0.9034 & 0.0801 \\
\hline \multirow{3}{*}{$\mathrm{Zn}(73)$} & PLS & $6^{a}$ & 0.9158 & 0.0018 & 0.9613 & 0.0012 \\
\hline & LS-SVM $^{\mathrm{d}}$ & $(81.3,3,389.1)^{b}$ & 0.9838 & 0.0009 & 0.9665 & 0.0012 \\
\hline & Lasso & $100^{c}$ & 0.9561 & 0.0013 & 0.9100 & 0.0019 \\
\hline \multirow{3}{*}{$B(62)$} & PLS & $6^{a}$ & 0.9579 & 0.0008 & 0.9432 & 0.0009 \\
\hline & LS-SVM $^{\mathrm{d}}$ & $(569.8,16,582.3)^{b}$ & 0.9857 & 0.0005 & 0.9569 & 0.0008 \\
\hline & Lasso & $70^{\mathrm{c}}$ & 0.9515 & 0.0009 & 0.9195 & 0.0011 \\
\hline
\end{tabular}

${ }^{a}$ is the parameter for PLS for number of latent variables LVs, ${ }^{b}$ is the parameter for LS-SVM for penalty parameters (c) and kernel function parameters $(\mathrm{g}){ }^{\mathrm{c}}$ is the parameter for Lasso for boundary value $\mathrm{t} ;{ }^{\mathrm{d}}$ means the best prediction model among three quantitative analysis methods for the specific element.

\section{Discussion}

For better univariate analysis, the emission lines without self-absorption and overlapping peaks should be chosen (Figure 2). The spectral emission line intensity is related to the element content. For the lower content elements, the emission line is often too weak, which makes it difficult to distinguish the sensitive emission line and may cause misjudgment. Therefore, content prediction of $\mathrm{Zn}$ and B, which are trace elements with relatively low amounts, was performed with multivariate analysis. However, univariate analysis for the nutritive elements obtained poor results. The matrix effect in complex PN samples has a major responsibility for the poor results.

Matrix effects come from the complex plant tissue including differences in chemical compositions and physical properties of plant tissue such as hardness, roughness, porosity, and density [28]. 
Matrix effects also relate to optical and plasma properties that influence the ratio of a given emission line to the abundance of the element producing that line [27]. Table 2 shows more than 90 emission lines with intensity values being above 1000 a.u. The LIBS spectra contain not only sensitive emission lines of the target elements, but also a large amount of other elements' information containing matrix effects' information. Figure 3 also indicates that multiple emission lines of other correlated elements and background variables may contribute to the prediction of target elements. Univariate analysis only considered the intensity of the sensitive emission line and lose of information related to the matrix effects. Therefore, univariate analysis is not an appropriate way to detect the nutrient elements' content in PN herbs.

Unlike univariate calibration, multivariate analysis performed well in Tables 4 and 5 and had the ability to excavate more multi-variables, including not only the useful information from sensitive emission lines of object element, but also the complex information from matrix effects, continuous background, and shot-to-shot fluctuation of the laser [17].

The effective variables are the critical point of whether to choose univariate or multivariate analysis. Full spectra had 22,036 variables including effective variables and vast ineffective variables, which inevitably resulted in model complexity and instability. After Lasso was selected, the effective LIBS variables reduced from 22,036 to 64, 73, 61, 66, 73, and 62 for $\mathrm{K}, \mathrm{Ca}, \mathrm{Mg}$, Fe, Zn, and B, respectively. The variable screening process could significantly reduce the number of variables in LIBS spectra for model input and mitigate noise or irrelevant information from background interference and matrix effects. The results of LS-SVM models based on the selected variables clearly demonstrated the merit of variable screening by Lasso. After the variable screening, the $R_{p}$ and RMSEP of the SVM models were greatly improved. Comparing Tables 4 and 5, LS-SVM models based on the selected variables by Lasso weights obtained the best prediction performance for $\mathrm{K}, \mathrm{Ca}, \mathrm{Mg}, \mathrm{Zn}$, and $\mathrm{B}\left(R_{p}=0.9546,0.9176\right.$, $0.9412,0.9665$, and 0.9569 , respectively). For Fe content prediction, Lasso based on full spectra got the best result with $R_{p}=0.9207$. The fitting plots for the best results for $\mathrm{K}, \mathrm{Ca}, \mathrm{Mg}, \mathrm{Fe}, \mathrm{Zn}$, and $\mathrm{B}$ are shown in Figure 4.
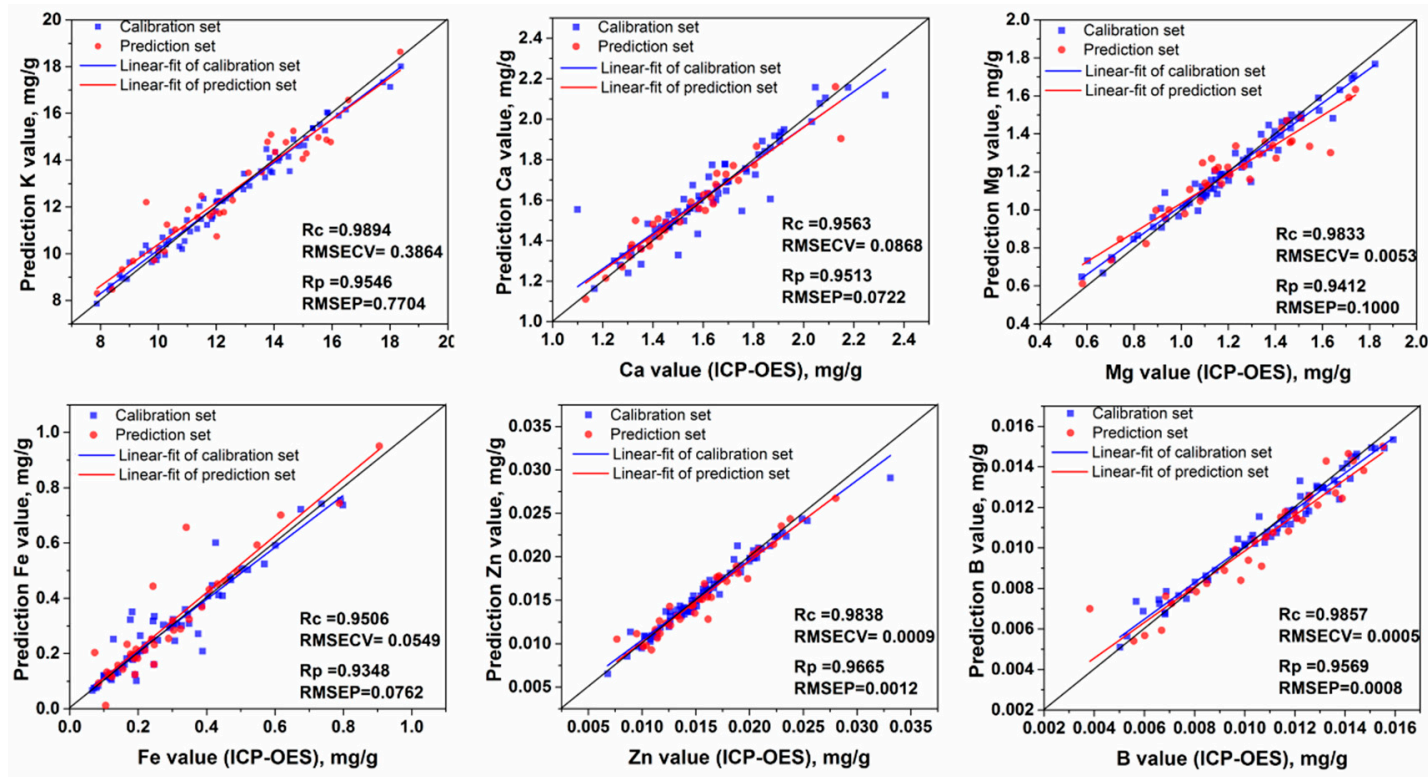

Figure 4. The best fitting plot of reference element content values and laser-induced breakdown spectroscopy (LIBS) measured element content values predicted by LS-SVM models (based on the selected variables) for $\mathrm{K}, \mathrm{Ca}, \mathrm{Mg}$, and $\mathrm{Zn}$ and Lasso models for Fe (based on full spectra).

PLS yielded comparable results in terms of accuracy both based on full spectra and the selected variables by Lasso weights. Therefore, PLS models could simultaneously account for changes in all these variables in the condition of changing environments and infinitely-variable sample chemistries. 
For $\mathrm{K}, \mathrm{Ca}, \mathrm{Mg}, \mathrm{Fe}, \mathrm{Zn}$, and $\mathrm{B}$ content detection of PN herbs, PLS may be better suited for this purpose. Lasso may be better for $\mathrm{Mg}$ and Fe content detection of PN herbs without more variable screening analysis, because the penalty method L1 selected variables from full spectral and the weights showed us the key emission lines used to quantify each element. The performance of LS-SVM models was limited by overfitting when confronted with full spectra. This may be a limitation of "large" LIBS variables and "small" sample sizes. Except Fe content analysis, the overfitting of LS-SVM models was alleviated after a reduction of more than $99.67 \%$ for variables by Lasso weights and showed the merit of the LS-SVM method dealing with the nonlinear relationship in the matrix effects and background interference. The LS-SVM models based on selected variables by Lasso were are the most suitable methods for prediction of $\mathrm{K}, \mathrm{Ca}, \mathrm{Mg}, \mathrm{Zn}$, and $\mathrm{B}$ content in PN herbs.

The quantitative analysis profiled the accuracy of LIBS combined with chemometrics, and our method also showed the ability of rapid detection for nutritive element content of PN herbs. Compared with the ICP-OES procedure, which needs more than a 150-min pretreatment containing weighting, adding other reagent, digesting, discharging acid, and diluting, the LIBS procedure needs less than $360 \mathrm{~s}$, because the grinding and tableting process of a PN sample is less than $250 \mathrm{~s}$ and LISB collecting of information of the pellet needs about $60 \mathrm{~s}$.

\section{Conclusions}

In this experiment, we demonstrated the rapid and accurate analysis of $\mathrm{K}, \mathrm{Ca}, \mathrm{Mg}, \mathrm{Fe}, \mathrm{Zn}$, and $\mathrm{B}$ content using our LIBS system based on $104 \mathrm{PN}$ samples from eight origins. More than 90 emission lines whose intensity values were above 1000 a.u. had their identities verified for PN samples. Univariate analysis based on sensitive emission lines could not meet the accuracy detection requirements of nutritive elements. For multivariable analysis, LS-SVM models based on the selected variables by Lasso weights for $\mathrm{K}, \mathrm{Ca}, \mathrm{Mg}, \mathrm{Zn}$, and $\mathrm{B}$ content detection obtained the best prediction performance with $R_{p}$ values of $0.9546,0.9176,0.9412,0.9665$, and 0.9569 and RMSEP of $0.7704 \mathrm{mg} / \mathrm{g}, 0.0712 \mathrm{mg} / \mathrm{g}$, $0.1000 \mathrm{mg} / \mathrm{g}, 0.0012 \mathrm{mg} / \mathrm{g}$, and $0.0008 \mathrm{mg} / \mathrm{g}$, respectively. The Lasso model based on full spectra obtained the best result with and $R_{p}$ value of 0.9348 and an RMSEP of $0.0726 \mathrm{mg} / \mathrm{g}$ for Fe content detection. PLS models performed good both with full spectra and the selected variables by Lasso weights. The Lasso weight plots provided direct information about effective variable's contribution to the quantitative analysis and can help researchers better understand the PN matrix.

LIBS technology combined with appropriate chemometric methods provided a fast, simple, and precise method for effective quantitative detection of nutrient elements in PN. However, further advances with more pharmaceutical analysis and other chemometric methods are still needed based on our study. Less and more effective variables should be discovered for the development of portable detection devices; ultimately, to provide a fast and accurate technique for laboratory analysis and online monitoring for Chinese herbal medicine quality and pharmaceutical analysis.

Author Contributions: Data curation, T.S., W.L., X.Z., and W.K.; formal analysis, T.S., W.K., F.L., and J.P.; funding acquisition, W.K., W.L., and F.L.; investigation, W.L., X.Z., and W.W.; methodology, T.S. and J.P.; project administration, W.L., W.K., and F.L.; resources, T.S., W.L., X.Z., and F.L.; supervision, F.L.; validation, T.S. and J.P.; visualization, J.P.; writing, original draft, T.S. and F.L.; writing, review and editing, T.S. and F.L.

Funding: This study was supported by the Natural Science Foundation of China (31671579), China Postdoctoral Science Foundation (2017T100431, 2016M600466), Applied Basic Research Foundation of Yunnan Province (2015FB205(-013)), and the Fundamental Research Funds for the Central Universities.

Acknowledgments: We thank the College of Life Science, Zhejiang University, for providing the ICP-OES.

Conflicts of Interest: The authors declare no conflict of interest. 


\section{References}

1. Ng, T.B. Pharmacological activity of sanchi ginseng (Panax notoginseng). J. Pharm. Pharmacol. 2006, 58, 1007-1019. [CrossRef] [PubMed]

2. Bai, J.; Mu, R.; Dou, M.; Yan, D.; Liu, B.; Wei, Q.; Wan, J.; Tang, Y.; Hu, Y. Epigenetic modification in histone deacetylase deletion strain of Calcarisporium arbuscula leads to diverse diterpenoids. N.a. Pharm. Sin. B 2018, 8, 687-697. [CrossRef]

3. Sun, S.; Wang, C.Z.; Tong, R.; Li, X.L.; Fishbein, A.; Wang, Q.; He, T.C.; Du, W.; Yuan, C.S. Effects of steaming the root of Panax notoginseng on chemical composition and anticancer activities. N.a. Chem. 2010, 118, 307-314. [CrossRef]

4. Yang, W.; Qiao, X.; Li, K.; Fan, J.; Bo, T.; Guo, D.A.; Ye, M. Identification and differentiation of Panax ginseng, Panax quinquefolium, and Panax notoginseng by monitoring multiple diagnostic chemical markers. N.a. Pharm. Sin. B 2016, 6, 568-575. [CrossRef]

5. Zhao, G.R.; Xiang, Z.J.; Ye, T.X.; Yuan, Y.J.; Guo, Z.X. Antioxidant activities of Salvia miltiorrhiza and Panax notoginseng. Food Chem. 2006, 99, 767-774. [CrossRef]

6. Fan, Z.Y.; Miao, C.P.; Qiao, X.G.; Zheng, Y.K.; Chen, H.H.; Chen, Y.W.; Xu, L.H.; Zhao, L.X.; Guan, H.L. Diversity, distribution, and antagonistic activities of rhizobacteria of Panax notoginseng. J. Ginseng Res. 2016, 40, 97-104. [CrossRef] [PubMed]

7. Toh, D.F.; New, L.S.; Koh, H.L.; Chan, E.C.Y. Ultra-high performance liquid chromatography/time-of-flight mass spectrometry (UHPLC/TOFMS) for time-dependent profiling of raw and steamed Panax notoginseng. J. Pharm. Biomed. Anal. 2010, 52, 43-50. [CrossRef] [PubMed]

8. Shan, S.M.; Luo, J.G.; Huang, F.; Kong, L.Y. Chemical characteristics combined with bioactivity for comprehensive evaluation of Panax ginseng C.A. Meyer in different ages and seasons based on HPLC-DAD and chemometric methods. J. Pharm. Biomed. Anal. 2014, 89, 76-82. [CrossRef] [PubMed]

9. Pytlakowska, K.; Kita, A.; Janoska, P.; Połowniak, M.; Kozik, V. Multi-element analysis of mineral and trace elements in medicinal herbs and their infusions. Food Chem. 2012, 135, 494-501. [CrossRef]

10. Maiti, R.; Rodriguez, H.G.; Kumari, A.; Sarkar, N. Macro and micro-nutrient contents of 18 medicinal plants used traditionally to alleviate diabetes in nuevo leon, northeast of Mexico. Pak. J. Bot. 2016, 48, 271-276.

11. Maiti, R.; Rodriguez, H.G.; Degu, H.D.; Kumari, C.A.; Sarkar, N.C.; Leon, F.D.C.F.U.A.D.N. Macro and micronutrients of 44 medicinal plant species used traditionally in nuevo leon, Mexico. Int. J. Bio-resource N.A. Manag. 2016, 7, 1054-1062. [CrossRef]

12. McCall, K.A.; Huang, C.C.; Fierke, C.A. Function and mechanism of zinc metalloenzymes. J. Nutr. 2000, 130, 1437S-1446S. [CrossRef] [PubMed]

13. Wang, B.H.; Yu, X.J.; Wang, D.; Qi, X.M.; Wang, H.P.; Yang, T.T.; Xu, X.H. Alterations of trace elements (Zn, $\mathrm{Se}, \mathrm{Cu}, \mathrm{Fe}$ ) and related metalloenzymes in rabbit blood after severe trauma. J. Elements Med. Boil. 2007, 21, 102-107. [CrossRef] [PubMed]

14. Karadaş, C.; Kara, D. Chemometric approach to evaluate trace metal concentrations in some spices and herbs. Food Chem. 2012, 130, 196-202. [CrossRef]

15. Tokalığlu, Ş. Determination of trace elements in commonly consumed medicinal herbs by ICP-MS and multivariate analysis. Food Chem. 2012, 134, 2504-2508. [CrossRef]

16. Mirosławski, J.; Paukszto, A. Determination of the Cadmium, Chromium, Nickel, and Lead Ions relays in selected polish medicinal plants and their infusion. Biol. Trace Elem. Res. 2018, 182, 147-151. [CrossRef]

17. Peng, J.; He, Y.; Ye, L.; Shen, T.; Liu, F.; Kong, W.; Liu, X.; Zhao, Y. Moisture influence reducing method for heavy metals detection in plant materials using laser-induced breakdown spectroscopy: A case study for chromium content detection in rice leaves. Anal. Chem. 2017, 89, 7593-7600. [CrossRef] [PubMed]

18. Peng, J.; Ye, L.; Shen, T.; Liu, F.; Song, K.; He, Y. Fast determination of copper content in tobacco (Nicotina tabacum L.) leaves using laser-induced breakdown spectroscopy with univariate and multivariate analysis. Trans. ASABE 2018, 61, 821-829. [CrossRef]

19. Peng, J.Y.; Liu, F.; Shen, T.T.; Ye, L.H.; Kong, W.W.; Wang, W.; Liu, X.D.; He, Y. Comparative study of the detection of Chromium content in rice leaves by $532 \mathrm{~nm}$ and $1064 \mathrm{~nm}$ laser-induced breakdown spectroscopy. Sensors 2018, 18, 621. [CrossRef] 
20. Juvé, V.; Portelli, R.; Boueri, M.; Baudelet, M.; Yu, J. Space-resolved analysis of trace elements in fresh vegetables using ultraviolet nanosecond laser-induced breakdown spectroscopy. Spectrochim. N.a. N.a. B: At. Spectrosc. 2008, 63, 1047-1053. [CrossRef]

21. Liu, F.; Wang, W.; Shen, T.; Peng, J.; Kong, W. Rapid identification of Kudzu powder of different origins using laser-induced breakdown spectroscopy. Sensors 2019, 19, 1453. [CrossRef] [PubMed]

22. Braga, J.W.B.; Trevizan, L.C.; Nunes, L.C.; Rufini, I.A.; Santos, D.; Krug, F.J. Comparison of univariate and multivariate calibration for the determination of micronutrients in pellets of plant materials by laser-induced breakdown spectrometry. Spectrochim. Acta Part B: At. Spectrosc. 2010, 65, 66-74. [CrossRef]

23. De Carvalho, G.G.A.; Moros, J.; Santos, D.; Krug, F.J.; Laserna, J.J.; Santos, J.D. Direct determination of the nutrient profile in plant materials by femtosecond laser-induced breakdown spectroscopy. Anal. Chim. Acta 2015, 876, 26-38. [CrossRef] [PubMed]

24. Gilon, N.; El-Haddad, J.; Stankova, A.; Lei, W.; Ma, Q.; Motto-Ros, V.; Yu, J. A matrix effect and accuracy evaluation for the determination of elements in milk powder LIBS and laser ablation/ICP-OES spectrometry. Anal. Bioanal. Chem. 2011, 401, 2681-2689. [CrossRef] [PubMed]

25. He, Y.; Liu, X.; Lv, Y.; Liu, F.; Peng, J.; Shen, T.; Zhao, Y.; Tang, Y.; Luo, S. Quantitative analysis of nutrient elements in soil using single and double-pulse laser-induced breakdown spectroscopy. Sensors 2018, 18, 1526. [CrossRef] [PubMed]

26. Zhang, Y.; Li, Y.; Gu, Y.H.; Guo, H.; Li, N. LIBS quantitative analysis of $\mathrm{Cr}$ and $\mathrm{Ni}$ in iron alloys with support vector machine (SVM). Spectrosc. Spectr. Anal. 2016, 36, 2244-2248.

27. Dyar, M.D.; Carmosino, M.L.; Breves, E.A.; Ozanne, M.V.; Clegg, S.M.; Wiens, R.C. Comparison of partial least squares and lasso regression techniques as applied to laser-induced breakdown spectroscopy of geological samples. Spectrochim. Acta Part B: At. Spectrosc. 2012, 70, 51-67. [CrossRef]

28. Liu, F.; Shen, T.T.; Kong, W.W.; Peng, J.Y.; Zhang, C.; Song, K.L.; Wang, W.; Zhang, C.; He, Y. Quantitative analysis of cadmium in tobacco roots using laser-induced breakdown spectroscopy with variable index and chemometrics. Front. Plant Sci. 2018, 9, 1316. [CrossRef]

29. Divya, B.; Harish, S.; Ramaswamy, K.; Kishorebabu, M.; Govindaiah, R.; Rambabu, U.; Munirathnam, N.R.; Raju, N. Estimation of $\mathrm{Cd}, \mathrm{Pb}$ and flame retardants in electric mosquito bat using EDXRF, ICP-OES, AAS and GC-MS. Int. J. Environ. Sci. Technol. 2017, 14, 2603-2612. [CrossRef]

30. Yang, W.; Li, B.; Zhou, J.; Han, Y.; Wang, Q. Continuous-wavelet-transform-based automatic curve fitting method for laser-induced breakdown spectroscopy. Appl. Opt. 2018, 57, 7526-7532. [CrossRef]

31. Tripathi, M.M.; Eseller, K.E.; Yueh, F.-Y.; Singh, J.P. Multivariate calibration of spectra obtained by laser induced breakdown spectroscopy of plutonium oxide surrogate residues. Spectrochim. Acta Part B: At. Spectrosc. 2009, 64, 1212-1218. [CrossRef]

32. Liu, F.; Ye, L.; Peng, J.; Song, K.; Shen, T.; Zhang, C.; He, Y. Fast detection of copper content in rice by laser-induced breakdown spectroscopy with uni- and multivariate analysis. Sensors 2018, 18, 705. [CrossRef] [PubMed]

33. Zhang, C.; Wang, Q.; Liu, F.; He, Y.; Xiao, Y. Rapid and non-destructive measurement of spinach pigments content during storage using hyperspectral imaging with chemometrics. Measurement 2017, 97, 149-155. [CrossRef]

34. Vapnik, V.N. The Nature of Statistical Learning Theory; Springer-Verlag: Berlin/Heidelberg, Germany, 1995.

35. Liu, F.; He, Y. Use of visible and near infrared spectroscopy and least squares-support vector machine to determine soluble solids content and $\mathrm{pH}$ of cola beverage. J. Agric. Food Chem. 2007, 55, 8883-8888. [CrossRef] [PubMed]

36. Wang, T.; He, M.; Shen, T.; Liu, F.; He, Y.; Liu, X.; Qiu, Z. Multi-element analysis of heavy metal content in soils using laser-induced breakdown spectroscopy: A case study in eastern China. Spectrochim. Acta Part B: At. Spectrosc. 2018, 149, 300-312. [CrossRef]

37. Ytsma, C.R.; Dyar, M.D. Effects of univariate and multivariate regression on the accuracy of hydrogen quantification with laser-induced breakdown spectroscopy. Spectrochim. Acta Part B: At. Spectrosc. 2018, 139, 27-37. [CrossRef]

38. Li, X.; Wang, Z.; Lui, S.L.; Fu, Y.; Li, Z.; Liu, J.; Ni, W. A partial least squares based spectrum normalization method for uncertainty reduction for laser-induced breakdown spectroscopy measurements. Spectrochim. Acta Part B: At. Spectrosc. 2013, 88, 180-185. [CrossRef] 
39. Moncayo, S.; Manzoor, S.; Rosales, J.; Anzano, J.; Caceres, J. Qualitative and quantitative analysis of milk for the detection of adulteration by Laser-induced breakdown spectroscopy (LIBS). Food Chem. 2017, 232, 322-328. [CrossRef]

40. Bricklemyer, R.S.; Brown, D.J.; Turk, P.J.; Clegg, S.M. Improved intact soil-core carbon determination applying regression shrinkage and variable selection techniques to complete spectrum laser-induced breakdown spectroscopy (LIBS). Appl. Spectrosc. 2013, 67, 1185-1199. [CrossRef]

Sample Availability: Samples of the Panax notoginseng are not available from the authors.

(C) 2019 by the authors. Licensee MDPI, Basel, Switzerland. This article is an open access article distributed under the terms and conditions of the Creative Commons Attribution (CC BY) license (http://creativecommons.org/licenses/by/4.0/). 